\title{
Oran R. Young, W. Bradnee Chambers, Joy A. Kim and Claudia ten Have (eds): Institutional Interplay: Biosafety and Trade
}

United Nations University Press, 2008, 198 pp

\author{
Keelie L. E. Murdock
}

Accepted: 10 May 2009/Published online: 7 June 2009

(C) The Author(s) 2009. This article is published with open access at Springerlink.com

Institutional interplay is an emerging concept and a practice with increasing significance and substantial consequence for contemporary governance systems. According to editors W. Bradnee Chambers, Joy A. Kim, and Claudia ten Have, interplay can be understood as "the phenomenon where one institution intentionally or unintentionally affects another" (pp. 3). The affordances provided by the concept have enabled the development of analytical themes and a vast array of sub-concepts with structuring properties. The study emerged in the early 1990s as an approach to understanding how international institutions work. It has enabled the development of knowledge about "boundary-problems" and how to manage tensions at the interfaces between institutional interests and agendas. It thus provides the means to understand the complex dynamics of institutional interaction. This is timely given that in the current state of globalization, international arrangements and regimes are contributing to increased institutional fragmentation and new patterns of interaction. Power has become more dispersed, politics more de-centralized, and boundaries more fluid. Thus, there is an unprecedented need for insights into how and why institutional interaction occurs and an evident requirement for practical knowledge to assist institutional actors with decision-making in the context of regime development and institutional coordination.

The editors of Institutional Interplay set out to bridge theory and practice by asking what theories of interplay can reveal about the relationship between the biosafety and the trade regimes and conversely, what the linkages between these regimes can reveal about institutional interplay. The strategy adopted required various scholars to analyze the case from the perspective of institutional interplay, albeit using different approaches, "as a means of drawing attention to similarities and differences among these approaches" (pp. 132). The editors expected that this approach would on the one hand contribute to the development of practical

K. L. E. Murdock ( $ه)$

Rathenau Instituut, Postbus 95366, 2509 CJ Den Haag, The Netherlands

e-mail: k.Murdock@rathenau.nl 
knowledge about the conditions, character and consequences of institutional interplay and effective ways to manage the interactions between institutions operating at the international level. On the other hand, there is an underlying expectation that the compilation of case-studies would contribute to theory-building and conceptual development. There are four studies in this volume, which aim to satisfy the research requirements. In addition, there is an introductory and concluding chapter written by the editors that interpret the results, a final chapter, which links the volume to its dedicatee, the late Konrad von Moltke, and a chapter that contains tributes to Konrad van Moltke from his past colleagues and collaborators. Taken together, the reader is introduced to the foundations of an emerging discipline and the frontiers and fault lines of biosafety in the global governance context.

This volume attempts to fill the knowledge gap about the dynamics of overlapping regimes by providing in-depth analyses of the linkages between the Biosafety Protocol under the Convention on Biological Diversity (CBD) and the World Trade Organization's (WTO) agreements on Sanitary and Phytosanitary measures (SPS) and Technical Barriers to Trade (TBT). This case is significant because these bodies represent crucial political structures through which important decisions are being made about the governance of Genetically Modified Organisms (GMO's). Exploring the overlap between them provides insight into the driving forces and motions of the decision-making process. The individual case-studies attempt to predict synergy and conflict by identifying cause-effect relationships between modes of interaction and the broader institutional context. They also identify opportunities for the effective mediation and management of differences between the priorities and preferences of the relevant members and institutions. This has the potential to facilitate practical decision-making. However, the results are too varied and abstract to offer a precise plan of action. A wide range of concepts are introduced, explained, and combined to enable overarching analyses. This sorting of critical attributes and experiences into different classes allows for expanded meanings and new understandings of the context under consideration but the usefulness of these concepts is obscured by a lack of conceptual disambiguation and a sufficient delineation of content. That is, without a common rule or reference point, the conceptual schemes overlap, making comparison difficult. Meanwhile, repetitive references to the same phenomena (including regime interactions, controversies, preambles and articles) occurs within each text and between them as each contributor strives to apply their approach to a limited context of interaction. In the concluding chapter, Oran Young assumes an apologetic tone and attempts to explain away the so-called "limits to taxonomy" (pp. 133). He attributes this problem to the complexity of the interactions between and within regimes, which introduces "methodological problems" for researchers and has "triggered a proliferation of conceptual distinctions" (Ibid). However, rather than mapping the overlapping and intersecting concepts, he regroups the elements of interplay into a set of competing categories with yet another explanation of the complexes. These intricacies are compounded by the limited index, which groups concepts under topics and does not include the names of quoted authors, which complicates efforts to search by concept and map their origins. 
In the introduction to the volume, Chambers et al. review the literature and identify the different types of linkages uncovered by scholars that have been influential to the development of the theoretical terrain. This introduction effectively prepares the reader for the conceptual overload that is to follow. The literature review introduces Oran R. Young's (ed.) taxonomy of interplay that includes, vertical, horizontal, functional, and political linkages and four institutional arrangements (embedded, nested, clustering, and overlapping). Standing in contrast to Young's analytical model is that of Olav Schram Strokke's model, which differentiates between diffusion, political spill-over, and normative, operational, utilitarian, and ideational interplay. Originating from the US and Norway, respectively, each scholar began with a similar aim and developed slightly different means to achieve it. Kristen Rosendal (also from the Fridtjof Nansen Institute in Norway) is credited with differentiating between the conditions for interplay on the basis of their effectiveness in the context of issue-based interactions and providing categories of effect (disruptive and supportive), characteristics of regimes (core and secondary aspects), and rule distinctions (regulatory and programmatic). This causal approach to the study of interplay spread to Germany where Sebastian Oberthür and Thomas Gehring adapted the distinction between synergistic and disruptive outcomes to the analysis of information flows between source institutions and target institutions, which are differentiated by the directionality and intentionality of institutional interaction to formulate a framework of interaction that illuminates the mechanisms of causality. Part II of this book utilizes these and other concepts to break the institutional interactions down into several layers of interplay. These chapters can be useful for readers interested in the conceptual schemes and how they can be applied. However, since it seems inevitable that an overview of the approaches and arguments will suffer from the same difficulties and dilemmas present in the work itself, this review will not attempt a thorough representation.

The introductory chapter by Aarti Gupta provides a sufficient overview of the dynamics of regime evolution and institutional decision-making. She sheds light on how the controversies between the biosafety and trade organizations have unfolded and how they have become concentrated on specific elements of discourse. This is an interesting addition to the volume because rather then identifying specific types of interplay and theorizing about the implications and causal effects, Gupta identifies areas of "potential regime interplay." This approach seems somewhat misdirected in contrast to the chapters in Part II of the volume, as though the author misunderstood the mission to apply concepts of interplay and instead identified concepts affecting it. However, including this chapter in the introduction provides a useful prelude to the difficult dilemmas perpetuated by the divide between the preferences of importing and exporting nations and how decisions made in one context spill-over into other contexts to influence the design, interpretation and implementation of global rules and regulations. For instance, the interplay between preferences for deregulated trade and enhanced safety, respectively, was at the heart of the "beef hormone" conflict in the early 1990s and the processes that emerged at this time have continued to shape GMO governance. The transatlantic conflict stemming from the European Unions hesitance to accept US beef products treated with a growth hormone into the regions market led to the negotiation of the WTO 
SPS and TBT Agreements, which aim to prevent protectionism by establishing international standards. The challenge for the WTO has since centered on finding acceptable definitions of the terms of the agreement, including "necessary" and "arbitrary" trade restrictions and criteria to qualify "science-based decisions" and "other legitimate factors" in food safety assessments. The Codex Alimentarius Commission's Ad Hoc Intergovernmental Task Force on Foods Derived from Biotechnology and the Biosafety Protocol under the CBD were established almost a decade later to manage some of the more complex issues associated with the global trade of GMO's and other products emanating from the application of biotechnology. The Biosafety Protocol has since been in conflict with the WTO over principles of risk analysis and risk management procedures, the basis for scientific decisionmaking under conditions of scientific uncertainty, information sharing obligations, and dispute settlement mechanisms. Accordingly, Gupta insightfully questions

whether global safety standards and guidelines... offer a way to reduce conflict and harmonize domestic regulation (as envisioned by the SPS Agreement) or whether they are inextricably caught up within the very same conflicts, with seemingly technical safety standards mirroring ongoing political conflicts in parallel global, regional and national forums (pp. 26).

There is a broad agreement among the contributors to this volume that the biosafety and trade regimes are co-evolving and the respective institutions are engaged in a complex process of boundary management. At the highest levels of abstraction, the institutional linkages between them are straightforward. The relationship between the Biosafety Protocol and the WTO's institutions governing trade in GMOs exists along the horizontal axis because they operate parallel to each other at the international level. Horizontal interplay involves institutions that are not locked into the same hierarchal structure, which enables reciprocal interaction. This suggests that decisions made in one context can resonate into the other and in turn affect the decision-making process. In the context of interactions between biosafety and trade, the horizontal linkage has engendered an overlap of socio-economic interests, jurisdictions, and membership. Meanwhile the linkage between these organizations and national implementing institutions exists on the vertical axis because they interact across levels of social organization and thus tend to occur in a top-down fashion. Vertical interplay has contributed to the development of tensions between the core values of the institutions and the state preferences for either trade regulations or trade liberalization. While these interactions have limited the potential for each organization and institution to carry out its objectives, they have also motivated actors to find workable compromises. ${ }^{1}$

The WHO's Biosafety Protocol appears to be at a disadvantage because since its emergence onto the international governance scene, it has been faced with the challenge of confronting the established trading system, its conventions, and its strongest supporters with its demands for environmental protection. However, as

\footnotetext{
1 Steve Charnovitz's concluding chapter entitled, "The WTO as an Environmental Agency" provides fresh insight into how these compromises may inevitably be realized by reframing the WTO as an environmental organization with the power and potential to managing tensions between the pursuit of economic wealth and environmental concerns.
} 
Oberthür and Gehring observe, "the case demonstrates a rather surprising strength of the seemingly weak biosafety regime vis-à-vis the supposedly much stronger WTO" (pp. 107). Thus, this case problematizes realist views of the place of material power in global politics and policy-making processes and highlights the influence of ideas and interactions on outcomes and output. It is perhaps unfortunate then that the relationship between material and ideational power is not explored and the influence of network formation remains latent. Nevertheless, it becomes clear through the course of this book that while there is still a lot of theoretical work to do to unite this promising field of study, the groundwork has been set for innovative analyses of institutional dynamics with the potential to solve some of the complex problems currently exacerbating our understandings of international relations and globalization.

Open Access This article is distributed under the terms of the Creative Commons Attribution Noncommercial License which permits any noncommercial use, distribution, and reproduction in any medium, provided the original author(s) and source are credited. 